\title{
Analysis and Design on Tourism Resources Management System Based on GIS
}

\author{
Shiwei Yuan \\ College of Finance and Trade, Bohai University, Jinzhou, 121013, China \\ swyuan1966@126.com
}

Keywords: GIS; tourism resources; management system; analysis and design

\begin{abstract}
Tourism resources has a very close relationship with geographical location, attractions distribution, tourism facilities, roads information are based on geographic coordinates. This paper Based on GIS which integrated MIS technology, provide technical support for system development. First, the design of the systems integration program by the MapInfo software, Map components, VC model library,.NET framework and database server configuration; then, use a structured analysis of data flow diagrams and data dictionary technology for user needs analysis; finally, systematic work process and software features as the basis for the system design process. The design content development software in this paper, has powerful data processing and spatial analysis capabilities, achieve the integration of numerous functions, could manage and tourism needs of all users of tourist information.
\end{abstract}

\section{Introduction}

Tourism resources management information system (TRMIS) is face to tourists, tourism operators and travel manager, in computer software and hardware's support, is a management information system integrated by tourism resource information collection, storage, processing, analysis, display and transmission functions. On the basis of geographic information system technology, fully use of GIS spatial data collection, storage, management, query and analysis functions, dynamic collection and efficient management for tourism resource information, achieve space analysis of tourism resource data, comprehensive evaluation, dynamic forecasting and other functions, and timely and accurate feedback to the system users. Object of TRMIS management include the natural tourism resources and cultural tourism resources of the tourism destination [1,2]. Function of the system cover all aspects of tourism resources information management, can achieve on tourism resources query, retrieve, statistics, choose the best route, chart printout and other functions. It plays an important role in query, investigation and management of tourism resources, tourism resources development and protection, tourism planning, tourism scenic environment carrying capacity and other aspects of tourism resource information. This paper Based on GIS technology which integrate MIS technology, provide support for the system development.

\section{System Integration Scheme}

Geographic information system (GIS) is a comprehensive discipline, combines with geography and cartography and remote sensing and computer science, has been widely applied in different fields, is a computer system which used for input, storage, query, analyze, and display the of geographic data.GIS is a tool that based on computer, can analyze and process spatial information, map and analysis the existence of phenomena and events on earth. Map of this unique visual effects and geographic analysis functions and he normal database operations are integrated into GIS technology.

GIS can be divided into five parts: people is the most important GIS component.GIS developer must define the various tasks to be performed in GIS, develop handle program. Skilled operators can usually overcome the lack of GIS software features, but the opposite situation cann't establish. The best software cann't compensate for the negative effect of the operator of the ignorant brought by GIS; data, accurate data available can affect the results of the query and analysis; hardware, 
hardware performance affect software for data processing speed, is whether easy to use and possible output; software, not only include GIS software, but also include a variety of databases, graphics, statistics, image processing and other programs; process, GIS require clearly definition, consistent method to generate the correct results verifiable $[3,4]$.

Under the control of the .NET Framework, use of component technology and model library technology, integrate MIS, MapInfo's MapX component and VC application analysis model, integrated program show in Fig. 1.

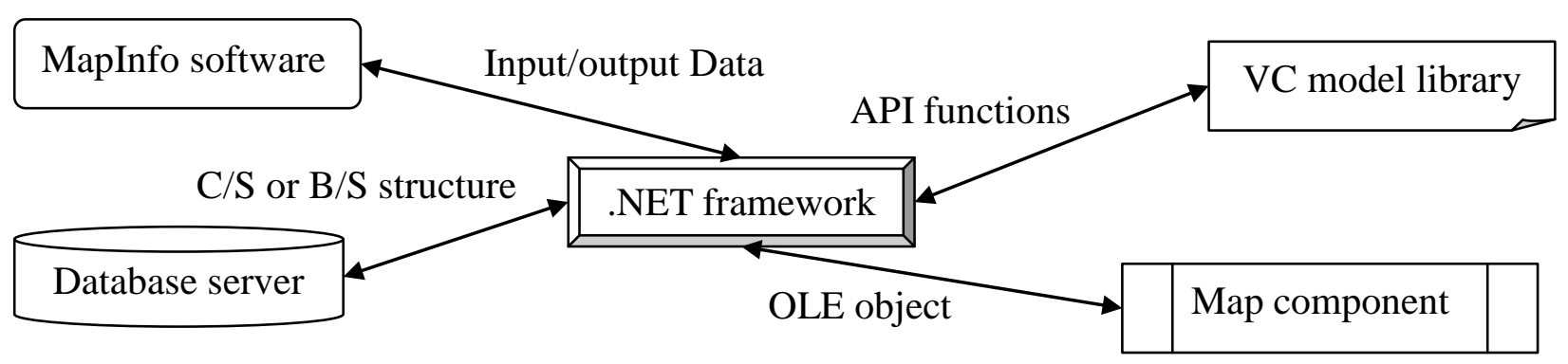

Fig. 1. System integration scheme

Using this program is mainly based on the following three considerations:

(1) MapX control provides developers with a fast, easy to use, function powerful map-based components. In the .NET visual development environment, MapX control add into the form just at the design stage, and be programmed to set properties, call methods, response time, can achieve data visualization, thematic analysis, geographic queries, geography coding and other rich GIS functionality. MapX define a class system, can effectively organize graphic elements, layers, attribute data and other objects.

(2) As a visual, object-oriented rapid MIS development tools, Microsoft .NET has a wide range of users, a variety of systems in many governments, the corporate sector are also based on .NET development..NET Object-oriented programming techniques, can help users to quickly establish a sound, in line with application requirements of the application model, reduce development cycles and development effort.

(3) VC model library is designed for the evaluation of tourism resources, VC is a well-known excellent low-level programming tool with many libraries have been forming used for programming and .NET API functions through the exchange of information with the model library, using this means of communication, so that evaluation of tourism resources tedious programming work are reduced, also improve the integration of performance.

\section{User Requirement Analysis}

Only through needs analysis, can the overall concept of software functionality and performance, describe as specific software requirements specification, thereby lay the foundation for software development. Method of needs analysis have many ways, including structured approach, object-oriented approach, prototyping approach, use cases modeling. This paper use structured approach, major tool of structured approach is the data flow diagram.

Data flow diagram (DFD) is a graphical technique, depict the flow of information and data from the input to the output of the process to move experienced transformation. Data flow diagram depict the system logic model, the figure don't have any physical element, only depict the flow of information in the system and deal with the situation. Data flow diagram generally include four elements: data flow, is the path of the data in the system spread, thus consists of a set of components consisting of fixed data; entity data source, on behalf of outside the system, can be a human, or other software system; processing, the data processing unit for receiving certain input data, process it, and produce an output; data storage, represent the static information storage, can represent documents, part of the file, and other elements of the database. Tourism resources management system is a tourism utility which solve the internal resources of the travel agency, have highly practical, not only can be used as a separate system, but also can be used as an important part 
of the tourism enterprise integrated business management system, and its management of the basic Regional thinking is based on information resources management tourist attractions, tourist hotels resources, tourism resources, restaurant, tourism and entertainment resources, tourism line resources. Data source is the user, including tourism staff, tour guides and travel personnel participating in several categories. System data flow diagram show in Fig. 2.

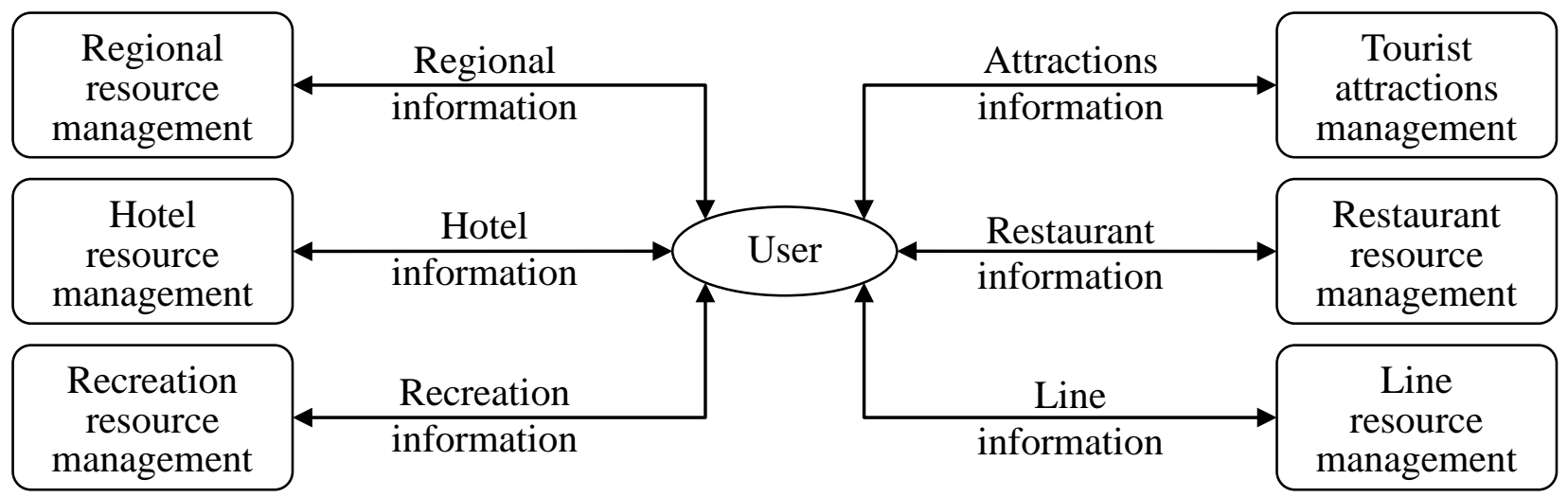

Fig. 2. Data flow diagram on tourism resource management system

Data Dictionary(DD) refer to data items, data structure, data flow, data storage, processing logic, external entities to define and describe, the purpose makes detailed instructions to the data flow diagram of the various elements. Data flow diagram and data dictionary together constitute a logical model of the system, there is no data dictionary data flow diagram isn't strict, there is no data flow diagram data dictionary is also difficult to play a role. Only data flow diagram and data dictionary which precisely define data flow diagrams together, can constitute the system specification. In Fig. 2,entry information include multiple data dictionary, limit length of this article, each class entry gives only one example.

\begin{tabular}{l|l} 
Name: User & Input data flow: User name and password \\
Alias: Tour guide user & Output data flow: Send event content \\
Location: User information table & Description: Explanation all information of the user
\end{tabular}

Definition: User $=$ User ID + Password + Name + Sex + Birth date + Country code + Guide card number + Address + Phone number + Graduated school + Learning professional.

Name: Tourist attractions

Alias: Scenic spot resources information

Location: System database
Data flow source: User (staff)

Data stream flow: User (all personnel)

Description: Description information on tourist attractions

Definition: Tourist attractions $=$ Attractions number + Attractions name + Website + Contact person + Phone number + Email + Communication address + Postalcode + Adult Price + Children fare + Area + Attractions Introduction + attractions pictures + Entry date and time.

Processing name: Attractions Management

Processing number: 1
Input data flow: Regional information

Output data flow: Tourist attractions

Processing logic: Management all information on tourist attractions, provide services for a variety of users. Manager maintain data, have an input, modify, delete permissions; tour guide tours, attractions pictures and other information to be updated; participate in tourism personnel can check all the information, and participate in online discussion.

\section{System Process Design}

The fundamental purpose of the detailed design phase is that determine how to specific achieve the system be required, that is to say, after this stage of the design work, should get an accurate description of the target system, in the coding phase can be directly translated into this description 
in some programming language written procedures. System process design show in Fig. 3.To protect system data security, user must enter a user name and password before using the system. System set up three-level users: ordinary user, advanced user and system administrator. The system can be used to set permissions based on the user's level. The highest privilege system administrator, can use all the modules, the lowest average user privileges, can only use the browse and search functions.

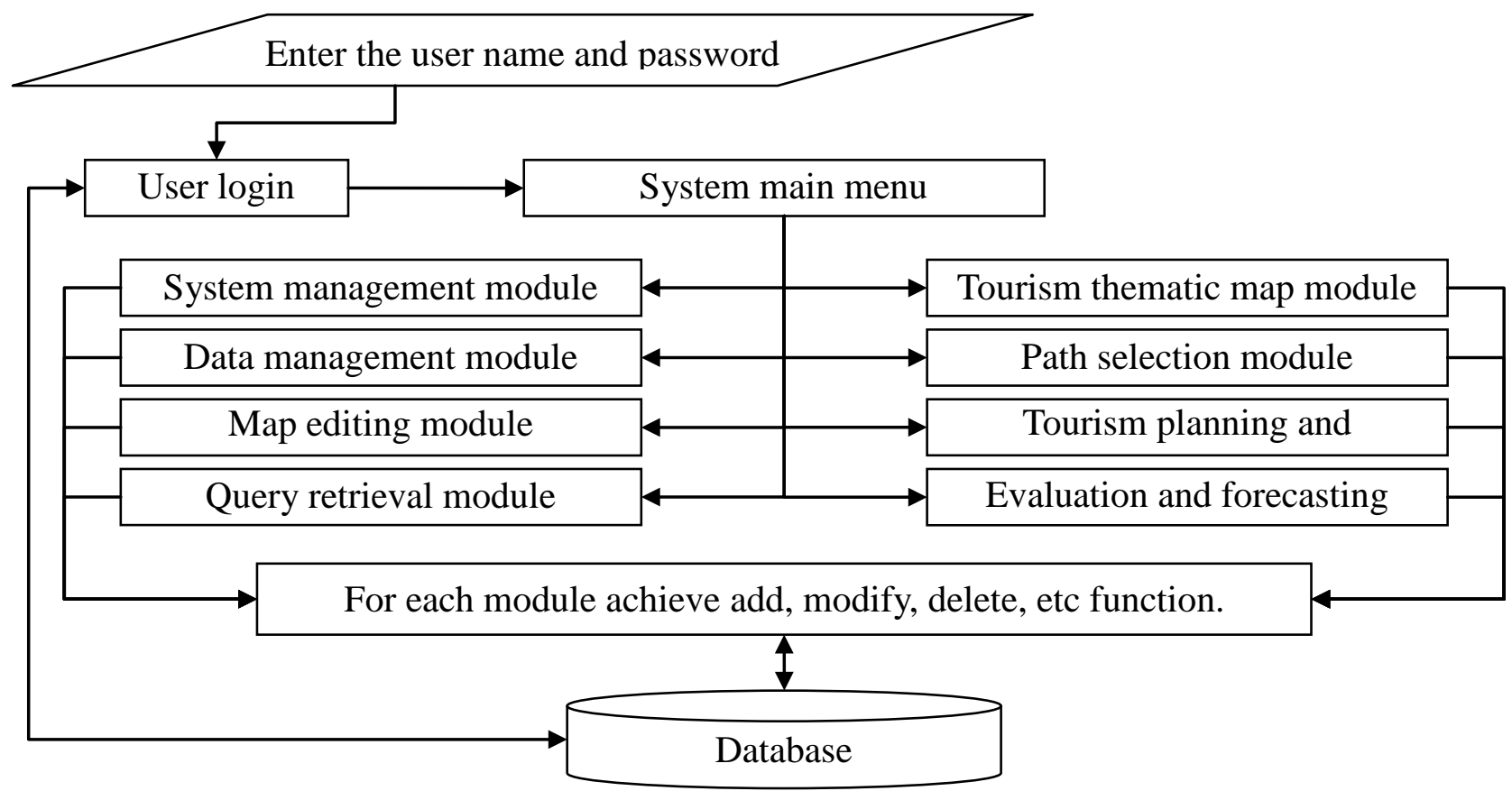

Fig. 3. Flow chart of system function

(1) System management module. A system administrator at the server log into the system can use the module, use to the user basic information management, user rights assignment, system configuration information management, backup and restore of the database, monitor for remote login user.

(2) Data management module. It is the most important part of GIS, provides storage, editing, search, query, calculation, display, ability to update the information space, and different from general database management system, not only the common attributes of the database management, but also managing spatial data, all attribute data are the description of a particular space object's position, with an intuitive and strong feature.

(3) Map editing module. Choose the appropriate command format layout in the Layout menu, such as layout, create scale, create compass, create legends, etc., after create the map layout view or map view can be displayed directly to the user, can also add printing equipment, will print out the information you need. Map roam, zoom, full inquiry positioning Fig operation, could be a new landscape marked on the map, the layer can be edited.

(4) Query retrieval module [5]. According to the user's requirement, from a variety of ways, such as property or graphics, images start of regional tourism resources, services, facilities and climatic conditions were flexible query retrieval. Query results can be used to retrieve other text, graphic and image display, with vivid and visual effects.

(5) Tourism thematic map module. GIS has strong graphics and text editing function, data maintenance is also very convenient, can greatly reduce the cost of showing, replace cumbersome process of traditional mapping, GIS graphics database is tiered storage, users can not only output total factor diagram, but also can require stratification or superposed output of various thematic maps based on the user.

(6) Path selection module. Including bus line inquiries and shortest path. Bus lines query, click on each bus line, the system will automatically search and showing flashes of the line and each site, click the site is displayed on the center position; shortest path, the shortest path between any two 
points on the road network in the solving and display the shortest path to solve it will be shown on the map, and displays the path length in the status area.

(7) Tourism planning and management. Tourism resources management is through people working and organized labor purposeful construction and management activities, changes in the physical form of tourism resources, convert it to the tourism product. Comprehensive analytical grade tourism resources, spatial and temporal distribution, size, and attractiveness of local socio-economic conditions, the use of system analysis function effectively carry out the development of the tourism master plan and detailed planning [6].

(8) Evaluation and forecasting module. By integrating with the mathematical analysis of the model, select the appropriate evaluation pointer, play space analysis function, evaluation model of tourism resources, tourism source markets and prediction, tourism resource capacity forecast model, capacity forecasting model for eco-tourism, economic development, tourism forecasting models "embedded" GIS in tourism management departments, can assist to make reasonable development decisions.

\section{Conclusion}

Emerging industry of Tourism, is accompanied by industrialization, globalization and information technology processes and growing, both products of economic and social development and progress, but also the flag of economic and social development and progress. Economic and social development, science and technology, real income growth, personal disposable leisure time increased, and people novelty, knowledge, seeking music, the desire to seek health enhancement, is the driving force behind the development of modern tourism. Tourism resource is one of the basic conditions for the development of tourism. Information has brought broad prospects for tourism development, through the application of information technology, the establishment of a unified tourism information database and operational platform, unify service development ideas, build a shared resource information database. This paper design tourist resources management information system based on GIS and MIS technical, not only have spatial characteristics of object, but also with a large number of attribute information, using application integration technology develop software, can achieve a number of functional integration, meet the management needs of all users and tourism travel information system.

\section{References}

[1] Atef A. Qaddah, Mohamed F. Abdelwahed, "GIS-based site-suitability modeling for seismic stations: Case study of the northern Rahat volcanic field, Saudi Arabia," Computers \& Geosciences, vol. 83, no. 10, pp. 193-208, 2015.

[2]

Baidu Encyclopedia

"Geographic

Information

System," http://baike.baidu.com/link?url=eP2nVTgucpboxhGydvSaLiBYWc7FJZywPVHTW1LgEifkD U8hDriV7uJ4up9ydqwXZuJAZSIMEpq9b6CTcp9zqSliFcqPiAVB7UhQ_WNVB, 2015-8-3.

[3] Y. Xiao, "Development of tourism resources information management system based on GIS," Master's degree of Central South University Of Forestry And Technology, 2004.

[4] X. L. Song, Y. Y. Tian, Y. Hou, "Technology and implementation method for the construction of tourism resource management information system," Mapping and spatial geographic information, vol. 36, no. 5, pp. 119-122, 2013.

[5] L. H. Zhuang, "The research and application of tourism resource management system solution," Micro computer application, vol. 28, no. 8, pp. 57-58, 2012.

[6] Romain Nouvel, Alessio Mastrucci, Ulrich Leopold, Olivier Baume, "Combining GIS-based statistical and engineering urban heat consumption models: Towards a new framework for multi-scale policy support," Energy and Buildings, vol. 107, no. 15, pp. 204-212, 2015. 